\title{
The influence of grooving corrosion on the strength of pipelines
}

\author{
Tatiana Zhilina ${ }^{1,}$, Konstantin Afonin ${ }^{1}$, Victor Mironov ${ }^{1}$, Artem Plotnikov ${ }^{1}$, and Anatoly \\ Shapoval ${ }^{1}$ \\ ${ }^{1}$ Tyumen Industrial University, 38, Volodarskogo Str., 625000, Tyumen, Russia
}

\begin{abstract}
The aim of the present work is to develop a model of grooving corrosion of the pipeline using APM WinMachine program. Corrosion is caused by chemical reaction of the metal with the environment (outside) and the transported product (inside) undergoing on the boundary of the metal and the media. This issue is widely investigated in multiple national and foreign studies; however, it is still relevant nowadays. This paper presents results of calculation of the stress-strain state of two segments of the pipeline with the given parameters. Engineering calculations were carried out using the finite element method. The stress maps were built based on the calculated models of pipelines.
\end{abstract}

\section{Introduction}

Corrosion causes serious damage, which is often associated with pipe leaks, damage of the soil integrity and in some cases it is accompanied by human losses [1,2].

The experience of pipelines operation reveals that pipelines made from carbon and lowalloy steel are mainly susceptible to the so-called grooving corrosion in the form of rills (or ditches) formed along the upper or lower outer generating lines of the pipe (Fig. 1). At the same time, the thickness of pipe walls along the rest of the perimeter almost does not decrease [3 - 7].
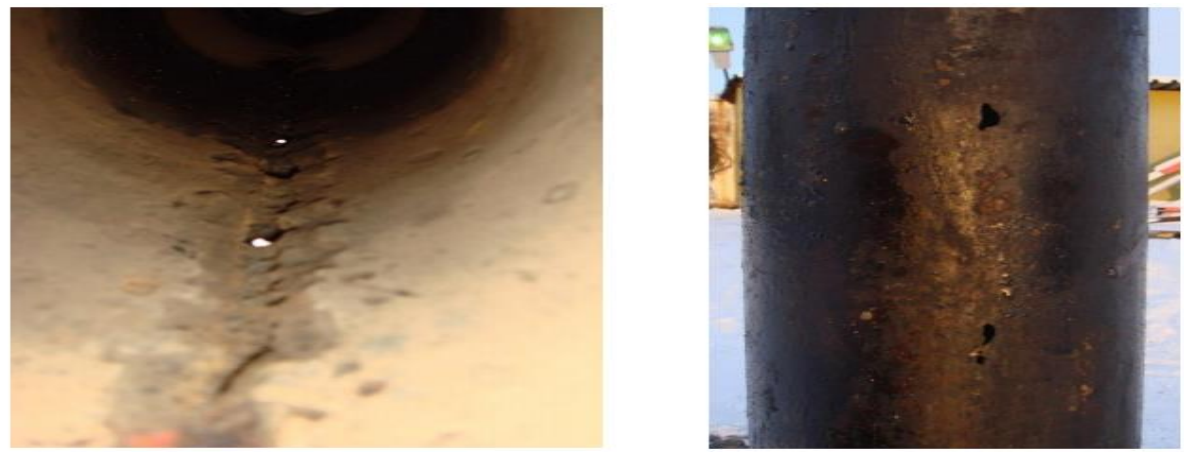

Fig. 1. Grooving corrosion.

\footnotetext{
*Corresponding author: ts-z@yandex.ru
} 


\section{Problem}

Grooving corrosion is one of the corrosion types that can occur both inside the pipeline and outside it. The inner part of the pipeline is constantly subjected to the impact of highly corrosive environment that can contain sand and mechanical admixtures. The outer part is exposed to the environmental impact, i.e. precipitation; weather conditions, etc. in the areas where thermal insulation is damaged [3 -7].

Moreover, grooving corrosion is aggravated by residual stresses of metallic origin acting in the pipe wall and stresses from the pressure of the pumped liquid. Under the influence of these factors the thinning of the pipeline takes place, which leads to pipe leaks and other more serious consequences [3-7].

\section{Methods and results}

Mathematical modeling of strength and resistance of pipelines can be carried out using various methodologies and parameters [8 - 11]; the present work is based on the finite element method.

Using the APM WinMachine program, the authors developed two models of a small section of the pipeline (Figs. 2, 3). Grooving corrosion was simulated on one of the pipelines in the form of a "ditch" along the inner surface of the pipeline; the other pipeline was not damaged.

Low-alloy heat-resistant steel $15 \mathrm{Kh} 5 \mathrm{M}$ (Russian grade $-15 \mathrm{X} 5 \mathrm{M}$ ) was used as the material of the pipeline, which is resistant to oxidation at temperatures up to $+600^{\circ} \mathrm{C}$, according to Russian State Standard GOST 20072-74.

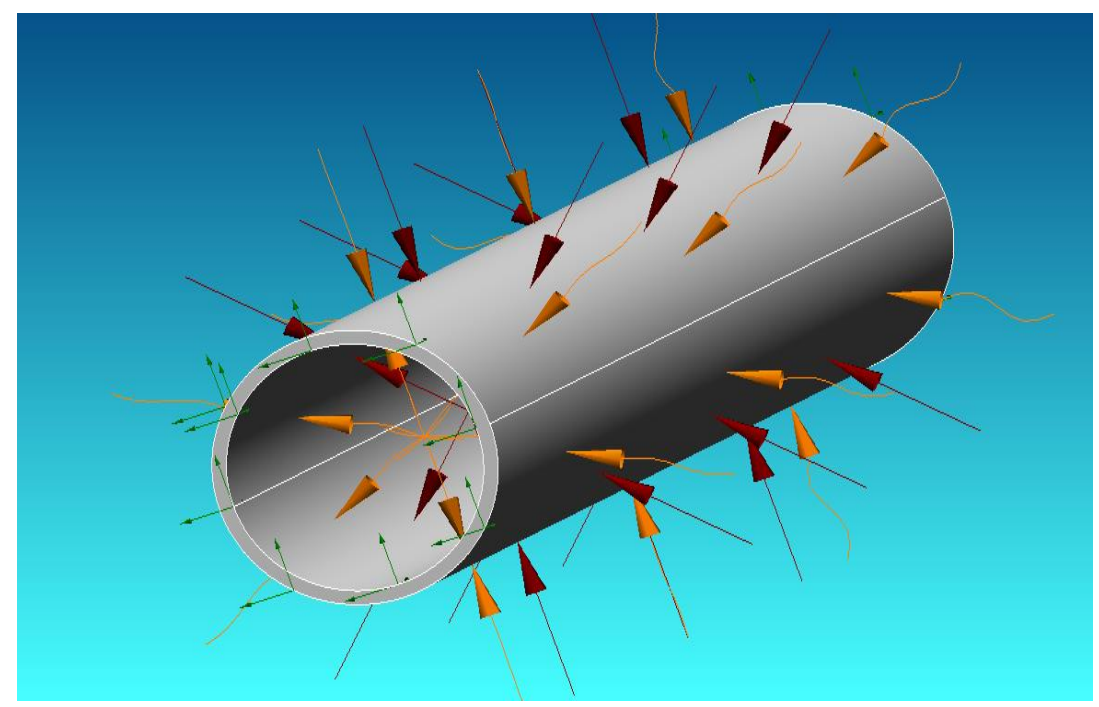

Fig. 2. Pipeline without defects (intact pipe). 


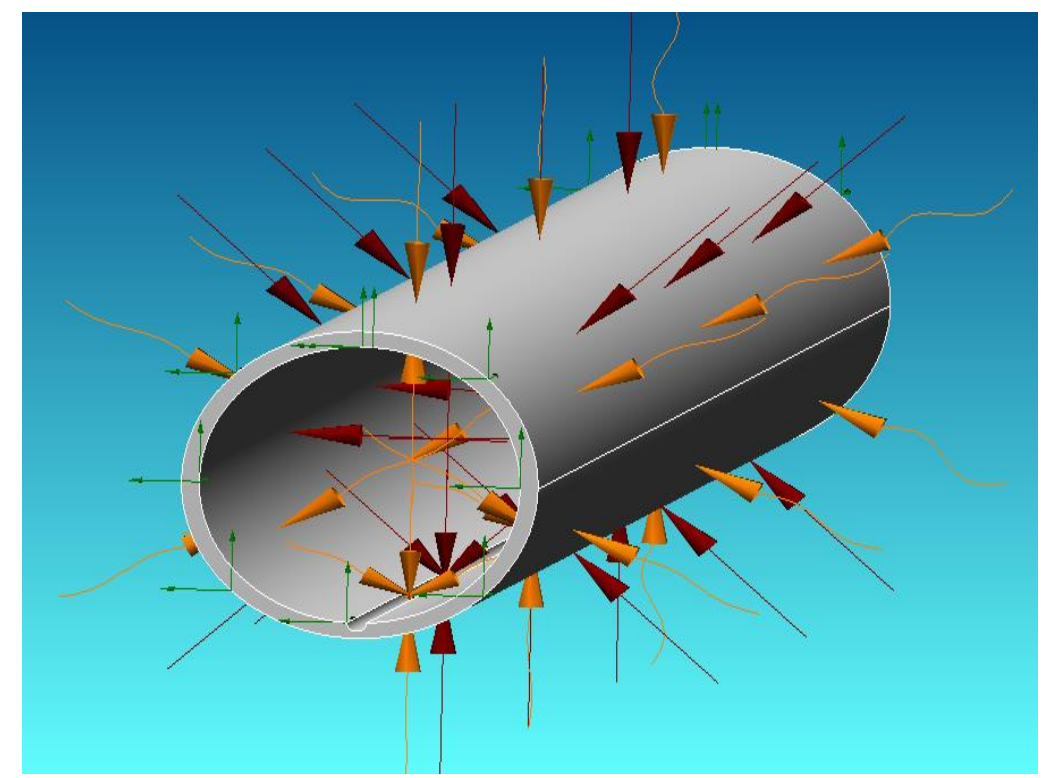

Fig. 3. Pipeline with simulation of corrosion in the form of a "ditch" (failed pipe).

Calculation of the stress-strain state was carried out considering the given parameters. Calculation results are shown in Table 1.

Table 1. Component specification.

\begin{tabular}{|l|c|}
\hline \multicolumn{1}{|c|}{ Component } & $\begin{array}{c}\text { The area of the pipeline, } \mathbf{1 . 5} \\
\text { m in length }\end{array}$ \\
\hline Material & $15 \mathrm{Kh} 5 \mathrm{M}$ \\
\hline Weight, $\mathrm{kg}$ & 6.9868062 \\
\hline Volume, $\mathrm{m}^{3}$ & 0.0008957 \\
\hline Compressive yield stress, $\mathrm{MPa}$ & 215 \\
\hline Young's modulus, $\mathrm{MPa}$ & 210000 \\
\hline Poisson's ratio & 0.3 \\
\hline Density, $\mathrm{kg} / \mathrm{m}^{3}$ & 7800 \\
\hline Thermal expansion coefficient, $1 /{ }^{\circ} \mathrm{C}$ & 0.000012 \\
\hline Thermal conductivity coefficient, $\mathrm{W} /\left(\mathrm{m} \times{ }^{\circ} \mathrm{C}\right)$ & 1 \\
\hline Ultimate compressive strength, $\mathrm{MPa}$ & 390 \\
\hline Limit of fatigue (initial), $\mathrm{MPa}$ & 199 \\
\hline Limit of fatigue (final), $\mathrm{MPa}$ & 98 \\
\hline
\end{tabular}

Table 2. Data on the load

\begin{tabular}{|c|c|c|}
\hline Item & Component & Load \\
\hline Pressure & Along the outer surface of the pipeline & $0.1 \mathrm{~N} / \mathrm{mm}^{2}$ \\
\hline Pressure & Along the inner surface of the pipeline & $4 \mathrm{~N} / \mathrm{mm}^{2}$ \\
\hline Temperature & of medium surrounding the pipeline & $200^{\circ} \mathrm{C}$ \\
\hline Temperature & of medium inside the pipeline & $150^{\circ} \mathrm{C}$ \\
\hline
\end{tabular}

The whole complex of the required engineering calculations was carried out in APM WinMachine using finite element method [12]. 
Finite element method is based on the fact that the model of a component or of the whole mechanism can be shown as a number of elements which are interconnected with each other at the top end points or in the nodal points along their boundaries. The sought-for values for each of the element can be found by means of their values in the nodes using some function. This function depends on the type of the used elements: a triangle or rectangle for plane problems and tetrahedron or parallelepiped for spatial problems (Figs. 4, 5) [12-19].

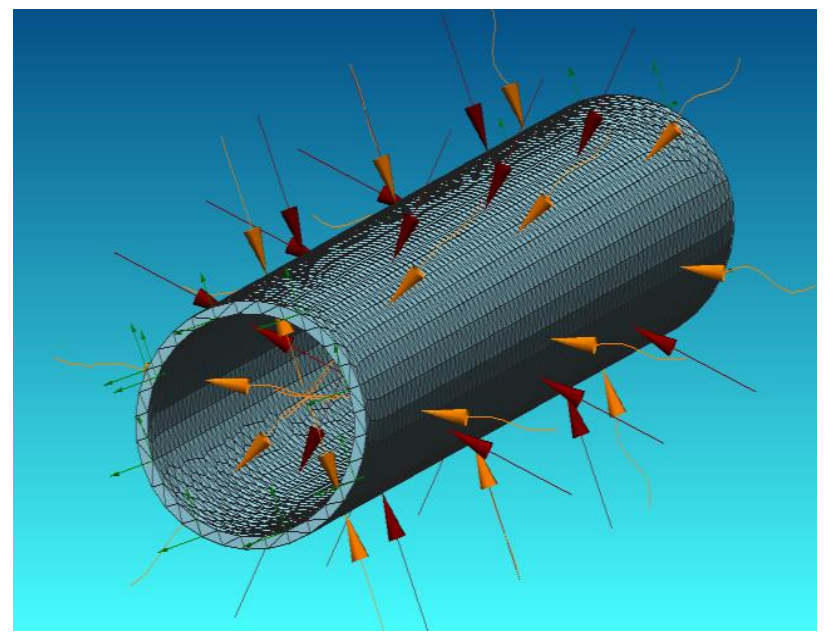

Fig. 4. Finite element mesh of the intact pipe.

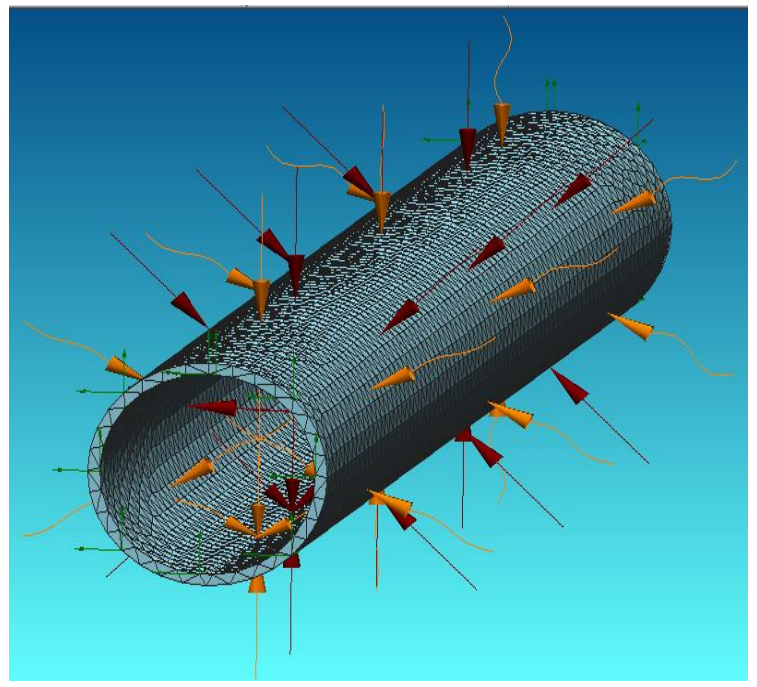

Fig. 5. Finite element grid of the failed pipe with simulation of grooving corrosion in the shape of a "ditch".

Regarding the calculations themselves, the program determines the von Mises criterion (the maximum von Mises stress or IV strength theory) which is based on Mises-Hencky theory, also known as the theory of energy [13].

The theory states that damages in plastic materials occur in the areas where the von Mises stress becomes equal to the ultimate stress. According to this theory, transition of metal to plasticity is associated with the value of potential distortion strain energy accumulated in a volume unit $[13,14]$. 
Mathematically the von Mises criterion is given as [13]:

$$
I I_{\Sigma \mathrm{D}}=C_{\mathrm{Y}}
$$

Or through the primary stresses:

$$
\left(\sigma_{\mathrm{I}}-\sigma_{\mathrm{II}}\right)^{2}+\left(\sigma_{\mathrm{II}}-\sigma_{\mathrm{III}}\right)^{2}+\left(\sigma_{\mathrm{III}}-\sigma_{\mathrm{I}}\right)^{2}=6 C_{\mathrm{Y}}
$$

In case of simple tension, it is easy to demonstrate that Formula 2 can be written as follows:

$$
\left(\sigma_{\mathrm{I}}-\sigma_{\mathrm{II}}\right)^{2}+\left(\sigma_{\mathrm{II}}-\sigma_{\mathrm{III}}\right)^{2}+\left(\sigma_{\mathrm{III}}-\sigma_{\mathrm{I}}\right)^{2}=6 C^{2} \mathrm{Y}
$$
shear:

The von Mises criterion can be also written using the value of yield stress under pure

$$
\left(\sigma_{\mathrm{I}}-\sigma_{\mathrm{II}}\right)^{2}+\left(\sigma_{\mathrm{II}}-\sigma_{\mathrm{III}}\right)^{2}+\left(\sigma_{\mathrm{III}}-\sigma_{\mathrm{I}}\right)^{2}=6 k
$$

The program provides a stress map for the two calculated models (Figs. 6, 7).

Table 3. Inertial properties of models.

\begin{tabular}{|l|c|c|}
\hline \multicolumn{1}{|c|}{ Item } & Value without defect & Value with defect \\
\hline Weight of the model, $\mathrm{kg}$ & 6.986806 & 6.909089 \\
\hline Center of gravity, $\mathrm{m}$ & $(-0.000001 ;-0.000003 ;$ & $(0.000019 ; 0.000318 ;$ \\
& $0.00011)$ & $0.000064)$ \\
\hline $\begin{array}{l}\text { Moment of inertia of the model relative } \\
\text { the mass center }\left[\mathrm{kg} \times \mathrm{m}^{2}\right]\end{array}$ & $(0.006226 ; 0.006221 ;$ & $(0.006183 ; 0.006139 ;$ \\
$0.327541)$ & $(-0 ;-0 ;-0)$ \\
\hline Reaction moment relative the mass & $(0 ;-0 ;-0)$ & $(0 ;-0 ;-0)$ \\
center [N×m] & $(0 ; 0 ;-0)$ & 0 \\
\hline Overall reaction of supports $[\mathrm{N}]$ & 0 & 0 \\
\hline The absolute value of reaction $[\mathrm{N}]$ & 0 & $\mathrm{SVM}$ \\
\hline The absolute value of moment $[\mathrm{N} \times \mathrm{m}]$ & & 13.062482 \\
\hline Von Mises Equivalent & $\mathrm{SVM}$ & 54.257986 \\
\hline - type, MPa & 11.676232 & \\
- minimum value & 34.799366 & \\
- maximum value & & \\
\hline
\end{tabular}

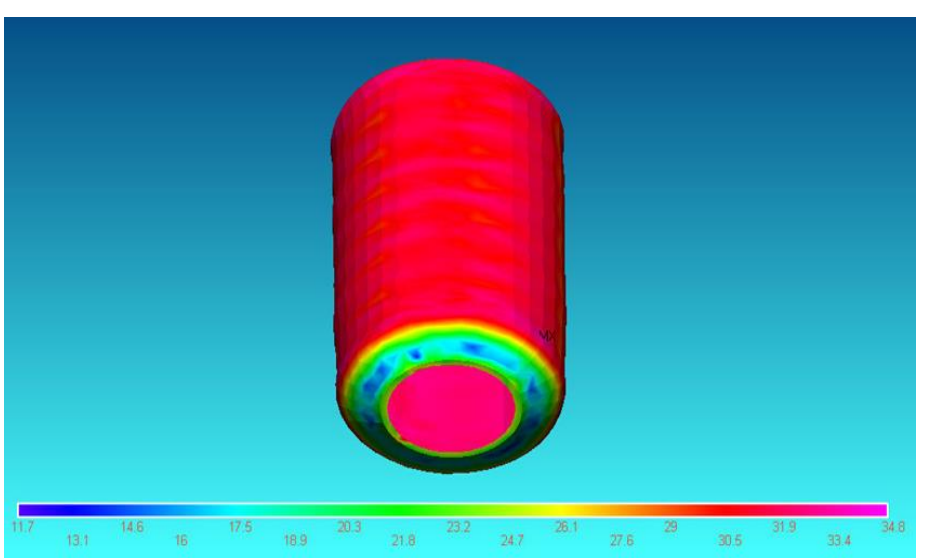

Fig. 6. A stress map of the intact pipe. 


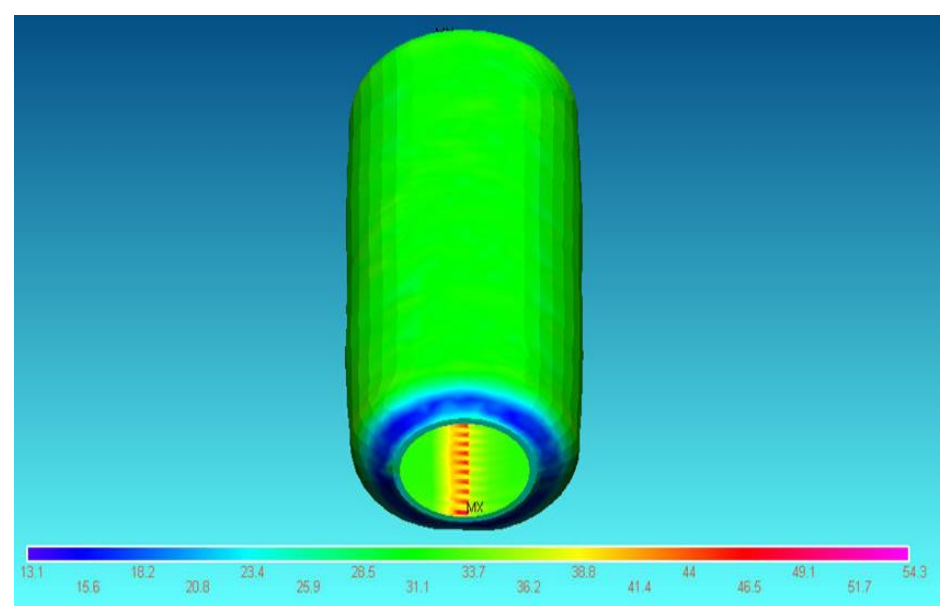

Fig. 7. A stress map of the failed pipeline with simulation of grooving corrosion in the shape of a "ditch".

Comparison of the stress maps of the intact and failed pipes shows that distribution of equivalent stresses in the other parts of the models (apart from the segment with a defect) differs insignificantly. In the place of a defect (corrosion) stress concentrator occurs and the stress level increases by 1.5 times compared to the stress map of intact pipe (from $34.79 \mathrm{MPa}$ in the intact pipe to $54.25 \mathrm{MPa}$ in the failed pipe). This leads to the pipeline break and involves a serious damage.

\section{Conclusion}

The results of work in APM WinMachine program and further processing of statistical data can be used for: [15]

- solving the complex objectives on consideration of the definite defect of the pipeline;

- making decisions on the reasonability of pipelines reconstruction;

- studying the stress-strain state of weld joints or the main nodes and components of the equipment;

- investigating and revealing the reasons of the occurred accidents, and also justification of making the corresponding amendments in the structure;

- conducting expertise with the aim of prolongation of the standard service life of pipelines.

\section{References}

1. L.A. Sosnovskiy, V.V. Vorobyev, A.A. Kostyuchenko, A.M. Bordovsky, World Tribology Congress III.1, 14 (2005) doi: 10.1115/wtc2005-63111

2. M. Rahman, , S. P. Murugan, , C. Ji, Y. J. Cho, J.Y. Cheon, Y.D. Park, Corrosion Science and Technology 17(3), 109 (2018) doi: 10.14773/cst.2018.17.3.109

3. A handbook of chemical industry worker 21. Chemistry and chemical technology, http://chem21.info/info/12298

4. V.V. Saveliev, A.N. Ivanov, Neftyanoe khozyaistvo 9 (1127), 120 (2017) doi: 10.24887/0028-2448-2017-9-120-122.

5. P.V. Burkov, S.P. Burkova, V.D. Samigullin, IOP Conference Series: Materials Science and Engineering 125, 012037 (2016) doi: 10.1088/1757-899X/125/1/012037 
6. R. Ashari, A. Eslami, M. Shamanian, S. Asghari, Journal of Materials Research and Technology (2019) doi: 10.1016/j.jmrt.2019.12.044

7. Hongwei Liu, Yuning Dai, Y. Frank Cheng, Arabian Journal of Chemistry (2019) https://doi.org/10.1016/j.arabjc.2019.11.006

8. O.V. Trifonov, V.P. Cherniy, Soil Dynamics and Earthquake Engineering 1(33), 54-62 (2012) doi: 10.1016/j.soildyn.2011.10.001

9. K. Bi, H. Hao, Engineering Structures 123.1 (2016) doi: 10.1016/j.engstruct.2016.05.022

10. P.V. Burkov, V.P. Burkov, S.P. Burkova, International Multidisciplinary scientific geoconference surveying geology and mining ecolory managment, SGEM 2018 (2018) doi: 10.5593/sgem2018/1.4/S06.036

11. P.V. Burkov, M.A. Filimonenko, S.P. Burkova, IOP conferences series: Earth and environmental science 43, 012044 (2016) doi: 10.1088/1755-1315/43/1/012044

12. Description of the program APM WinMachine, http://maintracker.org/forum/viewtopic.php?t=1097876, free

13. Von Mises Criterion (Maximum Distortion Energy Criterion.Engineer's edge (2018) https://www.engineersedge.com/material_science/von_mises.htm, free

14. F. Qingshan, Y. Bingchuan, C. Pengchao, A. Shirazic, Engineering Failure Analysis 106 (2019) doi: 10.1016/j.engfailanal.2019.104177

15. The experience of using APM WinMachine while conducting expert studies, http://sapr.ru/article/7192

16. R.B. Orlovich, R. Nowak, N.I. Vatin, V.V. Bespalov. Magazine of Civil Engineering. 2018. 82(6). Pp. 95-102. DOI:10.18720/MCE.82.9

17. I.N. Priadko, V.P. Mushchanov, H. Bartolo, N.I. Vatin, I.N. Rudnieva. Magazine of Civil Engineering. 2016. 65(5). Pp. 27-41. DOI:10.5862/MCE.65.3.

18. R.B. Orlovich, R. Nowak, N.I. Vatin, V.V. Bespalov. Magazine of Civil Engineering. 2018. 82(6). Pp. 95-102. DOI:10.18720/MCE.82.9.

19. D. Gura, Y. Dubenko, E. Dyshkant, A. Pavlyukova, G. Akopyan, IOP Conference Series: Earth and Environmental Science 403(1), 012184 (2019). DOI: 10.1088/1755$1315 / 403 / 1 / 012184$ 